\title{
Alianza entre aprendizaje y juego: gamificación como estrategia metodológica que motiva el aprendizaje del Inglés
}

\section{Gamification as a methodological strategy that motivates the learning of English creating an alliance between knowledge and game}

\author{
Lilia Patricia Rodríguez-Cajamarca \\ lilia.rodriguez@psg.ucacue.edu.ec \\ Universidad Católica de Cuenca, Azogues \\ Ecuador \\ https://orcid.org/0000-0002-3384-5558 \\ Darwin Gabriel Garcia-Herrera \\ dggarciah@ucacue.edu.ec \\ Universidad Católica de Cuenca, Azogues \\ Ecuador \\ https://orcid.org/0000-0001-6813-8100 \\ Claudio Fernando Guevara-Vizcaíno \\ cguevarav@ucacue.edu.ec \\ Universidad Católica de Cuenca, Cuenca \\ Ecuador \\ https://orcid.org/0000-0002-2341-6653 \\ Juan Carlos Erazo-Álvarez \\ jcerazo@ucacue.edu.ec \\ Universidad Católica de Cuenca, Cuenca \\ Ecuador \\ https://orcid.org/0000-0001-6480-2270
}

Recibido: 26 de abril de 2020

Revisado: 21 de mayo de 2020

Aprobado: 11 de junio de 2020

Publicado: 29 de junio de 2020 


\title{
RESUMEN
}

La investigación tuvo por objetivo determinar el impacto de la gamificación como estrategia metodológica empleada para motivar el aprendizaje del idioma inglés, permitiendo describir, analizar e interpretar la fortaleza que presentó la aplicación de esta didáctica en los grupos seleccionados. La metodología utilizada fue de tipo explicativa con diseño cuasi experimental, realizada a través de la plataforma Edutainment Mobbyt, que es una herramienta digital multimedia que permite realizar actividades didácticas gracias al uso de las TIC. Los estudiantes se motivaron a adquirir conocimientos nuevos del inglés mismos que lo utilizan en su vida cotidiana, didáctica de gran importancia para varios autores, quienes afirman la efectividad del uso de estrategias motivacionales en el aula que mejoran el nivel de suficiencia del inglés, sin embargo hay que analizar las desventajas que este puede provocar.

Descriptores: Lengua extranjera; enseñanza de idiomas; experimento educacional; innovación educacional. (Palabras tomadas del Tesauro UNESCO).

\begin{abstract}
The objective of the research was to determine the impact of gamification as a methodological strategy used to motivate the learning of the English language, allowing to describe, analyze and interpret the strength that the application of this didactic presented in the selected groups. The methodology used was of an explanatory type with a quasi-experimental design, carried out through the Edutainment Mobbyt platform, which is a digital multimedia tool that allows educational activities to be carried out thanks to the use of ICT. The students were motivated to acquire new knowledge of English themselves that they use it in their daily life, a didactic of great importance for several authors, who affirm the effectiveness of the use of motivational strategies in the classroom that improve the level of English proficiency, however You have to analyze the disadvantages that this can cause.
\end{abstract}

Descriptors: Foreign languages; Language instruction; educational experiments; educational innovations. (Words taken from the UNESCO Thesaurus). 


\section{INTRODUCCIÓN}

Para alcanzar potencialidad y participación en el mundo globalizado es necesario desarrollar habilidades sociales e intelectuales en diferentes áreas para afrontar a diferentes retos en el campo profesional, educativo y social, siendo de vital importancia el manejo de una segunda lengua como el inglés, misma que permita comunicarse de manera eficiente, dinámica y competitiva. Conviene subrayar que la rápida globalización demanda una interacción diaria con personas de diversas culturas, sobrepasando las barreras mundiales y dando impulso a la educación bilingüe en todos los países del mundo.

En este sentido, (Chávez Zambano Mirian, 2017), mencionan que en América Latina la enseñanza del inglés es todavía un reto, ya que existen dificultades para incorporar en los programas de estudio la enseñanza del idioma y lograr que los estudiantes al culminar un nivel de educación tengan el conocimiento y dominio de la lengua extranjera. A raíz de este precedente, Ecuador establece relaciones comerciales, política, sociales y culturales con otros países, donde el idioma inglés es el eje central en la mayoría de ellos, de ahí que se hace necesario fortalecer políticas educativas en el aprendizaje de una segunda lengua en la educación ecuatoriana, instituyendo reformas curriculares con metodologías que reflejen y respondan a las necesidades de los educandos (Ministerio de Educacación Ecuador, 2016).

A pesar de todos los esfuerzos realizados por el Ministerio de Educación, a lo largo de estos años, los resultados nacionales y locales no han sido alentadores porque un porcentaje mínimo alcanza un nivel de suficiencia del idioma al culminar el nivel medio. (Ortega-Auquilla \& Auccahuallpa-Fernandez, 2017), menciona que los estudiantes que continúan con sus estudios superiores se enfrentan a problemas porque no alcanzan un dominio del inglés, lo cual es una exigencia en la malla curricular especialmente en el nivel universitario, siendo un requisito indispensable aprobar un nivel de suficiencia para obtener el título de tercer nivel.

De donde se infiere que la gamificación motiva al estudiante a involucrarse de una forma dinámica en el aprendizaje, adoptándole como una didáctica innovadora que facilita el 
análisis y adquisición de contenidos de una forma significativa. Es por esto que el objetivo de la investigación fue determinar el impacto de la gamificación como estrategia metodológica empleada para motivar el aprendizaje del idioma inglés, permitiendo describir, analizar e interpretar la fortaleza que presentó la aplicación de esta didáctica en los grupos seleccionados.

\section{Referencial teórico}

Desde que el aprendizaje del inglés se convirtió de suma importancia en la educación actual, diversos métodos han sido creador para responder a las necesidades, sin dejar de lado un aspecto fundamental como la motivación en el aprendizaje del idioma extranjero, por ello se realizó una revisión de artículos investigativos de revistas, reportes, repositorios digitales y estudios experimentales que plantean didácticas que originen un aprendizaje significativo, optando por la gamificación como una estratégica de relevancia en el que hacer educativo, por cuanto a través de métodos lúdicos o juegos se consigue fortalecer habilidades lingüísticas y promover una participación activa de los educandos. (Marín Díaz, 2015) menciona que la educación actual conlleva a poner en práctica nuevos recursos que se han desarrollado a lo largo de las últimas décadas como wikis, flashmobs, infografías y más, como innovaciones dentro del aula. Entre éstos recursos tenemos a la gamificación, estrategia que potencia un aprendizaje significativo que, gracias al empleo de juegos, fomenta el desarrollo del proceso de enseñanzaaprendizaje efectivo, facilitando la cohesión, integración y motivación por el contenido. Cabe recalcar, para que la gamificación tenga éxito es importante considerar a la motivación como elemento principal, para que la adquisición de la segunda lengua tenga éxito (Briceño-de-Osorio, 2019).

(Cedeño Macías, 2017), menciona que existen diferentes didácticas que se pueden utilizar para motivar al estudiante al aprendizaje del inglés, la misma que se debe ajustar a las necesidades de cada uno de ellos, puesto que no existe un aprendizaje ni estilo puro. Dentro de estos modelos se manifiesta a los métodos comunicativos de (Richards Jack C., 1986) como: Content-Based Teaching, Task-based Instruction y Content 
Language Integrated Learning que afirman que este enfoque tiene una visión comunicativa utilizada para comunicarse y practicar la lengua.

Cabe recalcar que (Ortega Auquilla, Hidalgo Camacho, Siguenza Garzón, \& Cherres Fajardo, 2020) manifiestan que la motivación es la base para que se dé un aprendizaje significativo. Recalca que los factores intrínsecos y extrínsecos son los que determinen la adquisición de un conocimiento nuevo y ayudan al desarrollo de competencias lingüísticas. Por otra parte, (Gardner, 1985) define a la motivación como una combinación de variables complejas como actitudes y objetivos, los mismos que influyen para sostener la adquisición del lenguaje.

Hay que mencionar, además a (Bravo, Intriago, Holguín-Villafuerte, Garzon-Molina, \& Arcia-Ortega, 2017) quienes realizan aportes importantes sobre la motivación, misma que crea una interacción constante en el proceso pedagógico. Subrayando que para mantener el interés de los estudiantes es necesario potenciar su rendimiento, y que lo aprendido sea de utilidad en su vida diaria, en base a aptitudes que le permita afrontar a las dificultades que se presentan durante su vida. Es así que lo más importante para lograr una motivación en el proceso educativo es la utilización de didácticas activas e innovadoras que despierten el interés de los estudiantes de todas las edades.

Sin embargo, es importante conocer el origen de gamificación, misma que data desde el año 2008 cuando el británico (Deterding, 2011) presento este concepto en el campo empresarial con el propósito de construir modelos de producción. Mientras tanto que (Vanzin, Batista, \& Lopes da Silva, 2013) enfocan a la gamificación en el campo educativo con la aplicación de principios y elementos propios del juego en ambientes de aprendizaje con la intención de influir en la conducta, acrecentar la motivación y favorecer la participación de los estudiantes.

Continuando con el análisis de un estudio realizado por los químicos suecos Arvid Carlson, quienes mencionan a la dopamina, sustancia que permite el incremento de atención, motivación y memoria, lo cual es favorable en este proceso de enseñanza. Dicho en otras palabras, al presentar un tema nuevo a través de actividades lúdica o juegos, se manifiesta la dopamina en el sistema neuronal de los estudiantes, de esta 
manera ayuda a captar, adquirir y memorizar los nuevos conocimientos haciéndolos parte de su vida (Sanguineti, 2014).

Avanzando con más aportaciones sobre esta didáctica, (Chaves, 2019) define a la gamificación como una actividad lúdica que estimula la curiosidad y promueve la concentración, fortaleciendo de esta manera la memoria, la asimilación y procesamiento de léxico y estructuras sintácticas. En definitiva, los juegos en el aula incrementan la diversión y mejoran la atención favoreciendo al desarrollo cognitivo. No obstante, el uso de la gamificación en las aulas de clase debe ser limitada, su práctica no debe ser utilizada en todas las temáticas que se imparten, porque a la larga puede crear indiferencia (Caicedo-Coello, Vallejo-Valdivieso \& Moya-Martínez, 2020).

Habría que mencionar también a (Garcia-Gaitero, Garcia-Gaitero, \& Martin-Martin, 2018) quienes manifiestan que la gamificación es una instrumento interesante que a través del uso de la tecnología de la Información y Comunicación (TIC) potencia la estimulación de los estudiantes favoreciendo las relaciones interpersonales y de esta manera fomenta una participación constante en las diferentes actividades realizadas en clase. Conviene subrayar que los resultados obtenidos en su estudio demuestran que ha incrementado el grado de motivación de los estudiantes influyendo sobre el desarrollo de competencias lingüísticas.

Por otro parte, (Ruiz de la Hermosa, 2016) indica que, a las nuevas generaciones de estudiantes, conocidos como la generación nativa digital les gusta jugar la mayor parte del tiempo, su vida la desarrollan en un entorno computarizado predisponiéndose de esta manera a aprender y enfrentar a un aprendizaje a través de la mecánica del juego. De acuerdo a lo propuesto es importante integrar el juego en el proceso de enseñanzaaprendizaje, para de esta manera generar formas nuevas de instruir la teoría, la práctica, presentar los contenidos y evaluar. Sin duda alguna, esta metodología presentada surtió efectos positivos en este proceso de acuerdo a los resultados presentados, aumentó el agrado y la participación en clases, por ende, mejoró el rendimiento académico.

La metodología antes mencionada se desarrolló en la plataforma edutainmente mobbyt que tuvo su origen hace algunos años, plataforma tecnológica que invita a las personas 
a crear contenidos interactivos para educar. Ésta consta de un portal de juegos donde los contenidos creados pueden ser utilizados por cualquier docente o estudiante. Además, consta de una herramienta que facilita la creación de videojuegos. Ente las ventajas que posee esta plataforma podemos mencionar las siguientes: es gratuita y simple, funciona en cualquier dispositivo, no requiere de una instalación previa.

Esta plataforma ha ido ganado interés en centros educativos, universidades en toda Hispanoamérica. Asimismo, permite crear juegos de varios niveles mezclando las mecánicas disponibles, crea aulas y espacios virtuales. Los juegos más comunes de esta plataforma son trivias, tarjetas de memoria, coincidencias e historietas electrónicas, entre otros. Todos estos desafíos pueden ser agrupados secuencialmente dando lugar a un juego de multiniveles.

Una vez elegido el tipo de videojuego que se desea crear, el proceso es sencillo ya que se solo se tiene que seguir al asistente que ofrece la herramienta e ir completando la información. El primer lugar se tiene que elegir la mecánica del juego, inmediatamente se cargan los textos e imágenes a ser utilizados, a continuación, se crea la portada con un título, descripción u otra información para los usuarios. Finalmente, se publica para ser compartirlo en redes sociales, incluirlo en un blog o utilizarlo en el propio portal de la plataforma.

En cuanto a los beneficios que brinda a los estudiantes podemos describir los siguientes: permite aprender mientras se juega, asimismo promueven la superación personal a través de desafíos, igualmente permiten realizar experimentos repetidas veces sin costo, riesgo ni laboratorio especializados, finalmente, posibilitan aprender procedimientos tales como protocolos de seguridad y emergencia entre otros.

Seguidamente (Ortiz, Jordán, \& Agredal, 2018) también basan su teoría en elementos de diseño del juego en contextos educativos, generando de esta manera un proceso divertido, placentero y motivador. Añade, además que con la introducción de la mecánica y planteamiento del juego se involucra al estudiante de manera directa, considerando de este modo a la gamificación como una herramienta efectiva para mejorar el desarrollo de habilidades, destrezas, estimular la competencia y cooperación entre jugadores. 
Insistiendo que es importante que haya una relación controlada entre retos y capacidad para llevarlos a cabo este proceso, ya que estos elementos utilizado en exceso pueden causar poca o perdida de la motivación.

Prosiguiendo con aportaciones científicas sobre la mecánica del juego (Medina-Chicaiza \& Sanchéz Quishpe, 2018) consideran a esta metodológica como una técnica innovadora de enseñanza porque aporta una suma de estrategias idóneas, crea curiosidad e interés en los educandos quienes se sienten motivados en el proceso pedagógico. Aclarando de este modo, que la gamificación es una práctica pedagógica que tiene mucha importante dentro del quehacer educativo sobre todo con niños y adolescentes, grupo al que está enfocado este trabajo investigativo.

Asi mismo, (Garcia-Gaitero, Garcia-Gaitero, \& Martin-Martin, 2018) consideran a la gamificación como una herramienta pedagógica que ayuda a mejorar el aprendizaje de una forma significativa, además promueve una participación activa y a mantener el control en las diferentes actividades dentro del proceso enseñanza-aprendizaje. Siguiendo con la misma línea, (Figeroa-Flores, 2015) mientras tanto propone a la gamificación como una actividad motivadora que permite adquirir de forma dinámica una segunda lengua convirtiéndose de gran valor en la actualidad, ya que permite una comunicación directa con personas alrededor del mundo.

No obstante, luego del análisis de todos estos factores favorables, (Fernandez- Solo de Zaldivar, 2015) menciona algunas debilidades que se pueden presentar al utilizar esta didáctica. Señalando así, al factor económico porque no todos los juegos son de acceso libre, además la realización rápida y automática de tareas impiden la asimilación correcta del aprendizaje. La recompensa que reciben los estudiantes al realizar una tarea de forma inmediata, puede ser estimulantes al inicio, pero con el tiempo deja de tener valor. A más de esto, existe una linealidad que no permite enfocarse a la enseñanza personalizada, olvidándose de los intereses y estilos de aprendizaje de cada de los educandos.

Habria que decir tambien, (Llorens, et al., 2016), menciona otro aspecto negativo dentro de este proceso, al referirse al uso de video juegos, mismo que provoca en el estudiante una adicción e inmersión constante en esta actividad, lo cual no favorece a la adquisición 
de conocimiento nuevos, puesto que al momento de aprender no razona solo sigue reglas, instrucciones, es por ello que se ha vuelto imprescindible modificar a la gamificación con métodos innovadores través de plataformas e-learning que permita recolectar datos cualitativos y cuantitativos durante el proceso.

Considerando ahora la necesidad de actualizar a la gamificación (Johnson, et al., 2016) mencionan que a través de información recolectada en base a experiencias españolas sugieren actualizar a la gamificación en el ámbito educativos, de acuerdo al reporte obtenido del NMC Horizon Report (The New Media Consortium) demuestra que en los últimos años se ha generado la necesidad de un rediseño a esta didáctica que permita recolectar información automática con un esquema progresivo convirtiéndose en una innovación disruptiva basada en la tecnología.

Por lo tanto, como base de estudios investigatívos en los que se han encontrado aportes significatívos para la educación, se convierte en una necesidad importante re-plantear los métodos utilizados en las aulas ecuatorianas por actividades lúdicas, que es otro forma de aprender, sin dejar de lado las fortalezas y debilidades que esta tiene, ya que detrás de cada juego hay una serie de aprendizaje tanto de contenidos como de valores que motivan a los educandos a construir el conocimiento para que lo pueda utilizar en su vida futura

A partir de dicho referente, la presente investigación propone a la gamificación como una didáctica metodológica innovadora con la intención de motivar a los estudiantes a adquirir y practicar la lengua extrajera de una forma dinámica, despertando el interés y agrado por el inglés, y optimizar el desarrollo de las cuatro destrezas básicas hablar, escribir, leer y escuchar, mismas que son requeridas en el aprendizaje de un idioma. 


\section{MÉTODO}

La metodología utilizada fue de tipo explicativa con diseño cuasi experimental, realizada a través de la plataforma Edutainment Mobbyt, que es una herramienta digital multimedia que permite realizar actividades didácticas gracias al uso de las TIC. Esta plataforma es de acceso gratuito, segura para los menores de edad por cuanto los contenidos fueron creados por la docente y pueden ser vistos, aplicados, en cualquier hardware.

Se trabajó con temas gramaticales y vocabulario, la primera actividad utilizada fue un juego de trivias, en la que los estudiantes a través de imágenes seleccionaban la forma correcta del verbo para completar oraciones, además se puso en marcha un juego de tarjeta de memoria que a través de imágenes se asocia con una palabra o concepto. Otra actividad realizada a través del juego fue la creación de historietas simples, donde los estudiantes con el vocabulario proporcionado crean sus propias viñetas o cuentos. Finalmente se realizó un juego de coincidencias con el uso de dibujos y palabras nuevas. La investigación se desarrolló en la Unidad Educativa Particular "La Providencia" de la ciudad de Azogues, provincia del Cañar-Ecuador, institución educativa con una población estudiantil de 1400 alumnos, desde la educación inicial básica hasta bachillerato. Se realizó un muestreo aleatorio estratificado de 98 estudiantes que pertenecen los séptimos de básica.

La investigación es de cohorte longitudinal, se realizó una encuesta inicial con el propósito de conocer sobre métodos o estrategias favoritas de los estudiantes que motiven su aprendizaje del idioma. Seguidamente se realizó la experimentación con actividades lúdicas en las aulas seleccionadas en base a juegos creados en Mobbyt, concluyendo con una encuesta con la finalidad de comprobar el impacto que causó esta didáctica en el proceso pedagógico.

Las encuestadas aplicadas se realizaron de forma anónima con el fin de precautelar la identidad de los estudiantes, por cuanto son menores de edad comprendidas entre 9 a 13 años. La pre-encuesta se realizó en las aulas de clase el mes de febrero, instrumento que fue aplicado a 92 estudiantes de los 98 que forman parte de la propuesta didáctica, debido a la inasistencia de los estudiantes a la institución por diferentes razones. Mientras 
tanto que la post encuesta se hizo a través de Google Drive, enviando el link a la plataforma Idukay, misma que es utilizada por la institución para mantener una comunicación directa con los estudiantes, es de acotar que la fiabilidad del instrumento aplicado según el Alfa de Cronbach obtuvo un valor de 0,708.

Finalmente, los datos fueron examinados a través de un análisis descriptivo con la prueba $T$ de Student para muestra independiente del software SPSS (Startical Product and Service Solución) con el fin de obtener datos estadísticos fiables de la información recabada (Cordero-Naspud, Erazo-Álvarez, Narváez-Zurita, \& Cordero-Guzmán, 2020).

\section{RESULTADOS}

\section{Tabla 1}

Método preferido para motivar el aprendizaje del inglés

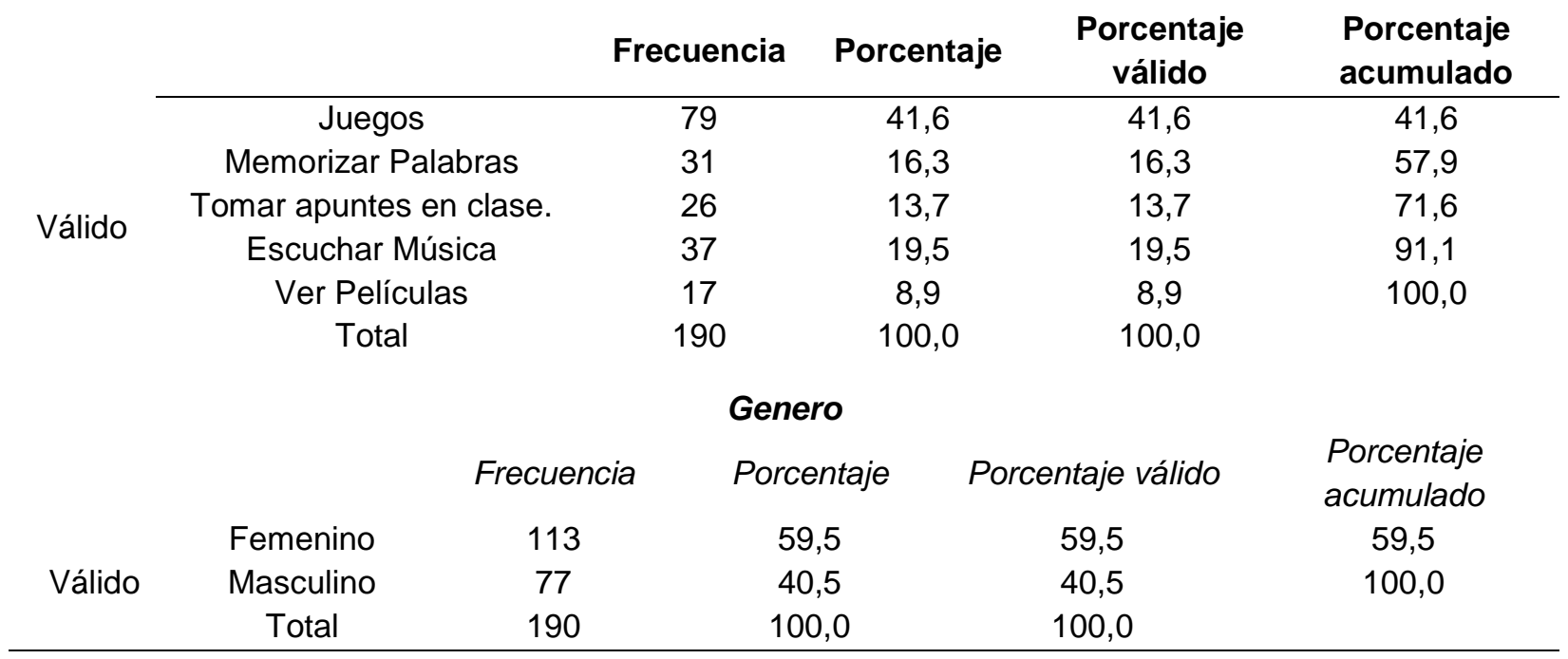

Fuente: Elaboración Propia

La tabla 1 demuestra que el $41.6 \%$ de los encuestados afirman que el método preferido para motivar el aprendizaje del inglés es el juego, a través de esta actividad se sienten motivados a adquirir conocimientos nuevos sobre la lengua extranjera. Continuando con el análisis se puede manifestar también, que la población femenina prefiere actividades lúdicas dentro del proceso educativo, confirmando de esta manera la teoría presentada 
Revista Arbitrada Interdisciplinaria KOINONIA

Año 2020. Vol V. ํ⒈ Especial Educación

Hecho el depósito de Ley: FA2016000010

ISSN: $2542-3088$

FUNDACIÓN KOINONIA (F.K). Santa Ana de Coro. Venezuela.

Luis Pillaga Guamán; Darwin Gabriel Garcia-Herrera; Nancy Marcela Cárdenas-Cordero; Juan Carlos Erazo-Álvarez

por (Medina-Chicaiza \& Sanchéz Quishpe, 2018), quienes afirman que la gamificación es una técnica innovadora que aporta una suma de estrategias idóneas, despierta el interés en el proceso formativo. Lo cual se lo puede comprobar con los resultados estadísticas obtenidas de la variable.

\section{Tabla 2}

Relación entre tres variables con sus medias obtenidas en el pre and post encuesta.

\begin{tabular}{|c|c|c|c|c|c|}
\hline & $\begin{array}{l}\text { Pretest and Post- } \\
\text { test }\end{array}$ & $\mathbf{N}$ & Media & $\begin{array}{l}\text { Desviación } \\
\text { estándar }\end{array}$ & $\begin{array}{l}\text { Media de } \\
\text { error } \\
\text { estándar }\end{array}$ \\
\hline $\begin{array}{l}\text { Las estrategias lúdicas } \\
\text { utilizadas por el docente le } \\
\text { han permitido adquirir }\end{array}$ & Pretest & 92 & 3,89 & 1,124 & 0,117 \\
\hline $\begin{array}{l}\text { conocimientos sobre la } \\
\text { lengua extranjera para } \\
\text { comunicarse. }\end{array}$ & Post- test & 98 & 4,38 & 0,753 & 0,076 \\
\hline $\begin{array}{l}\text { El docente emplea juegos } \\
\text { como estrategias }\end{array}$ & Pretest & 92 & 3,01 & 1,371 & 0,143 \\
\hline $\begin{array}{l}\text { didácticas para motivar la } \\
\text { enseñanza del inglés }\end{array}$ & Post- test & 98 & 3,93 & 1,018 & 0,103 \\
\hline Le interesa aprender & Pretest & 92 & 4,30 & 1,165 & 0,121 \\
\hline $\begin{array}{l}\text { inglés a través de } \\
\text { aplicaciones de juegos. }\end{array}$ & Post- test & 98 & 4,78 & 0,584 & 0,059 \\
\hline
\end{tabular}

\section{Prueba de muestras independientes}

Las estrategias lúdicas utilizadas por el docente le han permitido adquirir conocimientos sobre la lengua extranjera para comunicarse. Se asumen varianzas iguales asumen
El docente emplea juegos como estrategias didácticas para motivar la enseñanza del inglés Se asumen varianzas iguales varianzas iguales
Le interesa aprender inglés a través de aplicaciones de juegos.

$\begin{array}{ccc}\text { No se } & \text { Se } & \text { No se } \\ \text { asumen } & \text { asumen } & \text { asumen } \\ \text { varianzas } & \text { varianzas } & \text { varianzas } \\ \text { iguales } & \text { iguales } & \text { iguales }\end{array}$




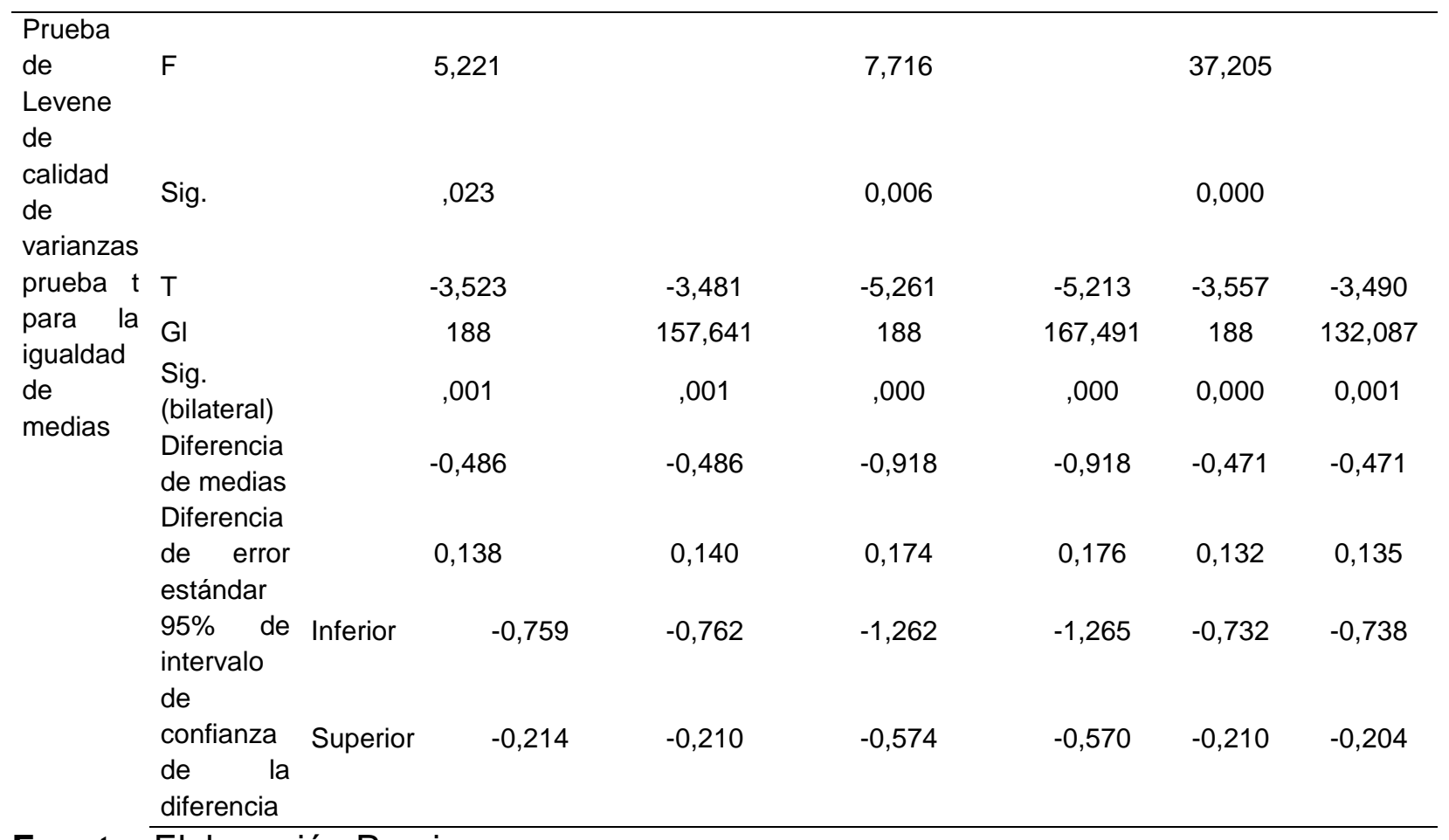

Fuente: Elaboración Propia.

\section{Hipótesis 1}

H0: La estratégica lúdica no depende para adquirir conocimientos sobre la lengua extranjera.

H1: La estrategia lúdica depende para adquirir conocimientos sobre la lengua extranjera. Los resultados que se encuentran en este rango, muestran que debido a que el porcentaje es menor al nivel de significancia la hipótesis es afirmativa, es decir que con el uso de estrategias lúdicas permite adquirir conocimientos nuevos sobre la lengua extranjera.

Esta hipótesis es afirmada por (Garcia-Gaitero, Garcia-Gaitero, \& Martin-Martin, 2018) quienes exponen que la didactica del juego es un instrumento interesante que estimula el aprendizaje y ayuda al desarrollo de competencias lingüísticas en cada uno de los estudiantes. No obstante, las afirmaciones de (Fernandez- Solo de Zaldivar, 2015), al considerar que con la utilizacion de las didacticas del juego impide una asimilacion correcta del aprendizaje, por cuanto existe una linealidad que no permite enfocarse a la 
ensenanza personanalizada dejando de lado las necesidades y estilos de aprendizaje de cada estudiante. Teoria que no concuerda con los resultados obtenidos, por cuanto se notó que un porcentaje superior adquirieron conocimientos a través de actividades lúdicas constantes lo cual los motivó.

\section{Hipótesis 2}

H0: El juego empleado por el docente como estrategia no depende para motivar la enseñanza del inglés.

H1: El juego empleado por el docente como estrategia depende para motivar la enseñanza del inglés.

Los resultados que se encuentran en este rango, muestran que debido a que el porcentaje es menor al nivel de significancia, la hipótesis es afirmativa, lo que significa que el docente al utilizar estrategias metodológicas como el juego motiva la enseñanza del inglés, pues autores como (Ruiz de la Hermosa, 2016), manifiesta que a los estudiantes actuales se les considera como la generación nativa digital, puesto que les gusta jugar todo el tiempo. Razón por la cual es importante integrar el juego en el proceso de enseñanza-aprendizaje generando de esta manera nuevas formas de presentar contenidos y evaluar.

\section{Hipótesis 3}

H0: El interés de aprender inglés no depende de las aplicaciones de juegos.

H1: El interés de aprender inglés depende de las aplicaciones de juegos.

Los resultados que se encuentran en este rango, muestran que debido a que el porcentaje es menor al nivel de significancia la hipótesis es afirmativa, lo que significa que al estudiante le interesa aprender inglés a través de aplicaciones del juego, (Ortiz, Jordán, \& Agredal, 2018) ratifíca la teoria basada en el juego dentro del proceso eduactivo. Considerando como una herramienta efectiva para mejorar habilidades y destrezas, estimular competencia y cooperancion entre los estudiante. Sin embargo, recomienda que debe existir un uso controlado por cuanto al recurrir en exceso de 
actividades lúdicas puede causar perdida de motivacion o desinterés. No obstante, los resultados demuestran que el uso constantes del juego les motiva a mejorar las habilidades y destrezas, no se considera la posibilidad de disminuir su uso, información que que no concuerda con la realidad.

En un análisis general se deduce que la gamificación dentro del aula es de gran importancia para los estudiantes, éste es utilizado para motivar al aprendizaje y en la mayoría de casos estimula la adquisición de conocimientos nuevos. Tal como lo demuestran las estadísticas, un porcentaje superior prefieren actividades lúdicas, juegos para involucrarse activamente en el aprendizaje de una nueva lengua.

Las estadísticas cumplen el objetivo de la investigación, los estudiantes consideran a la gamificación como una estrategia adecuada dentro del proceso de enseñanza aprendizaje porque este incitó al estudio del inglés, asignatura que ha sido considerada como difícil y de poco valor puesto que las didácticas utilizadas no han promovido su interés. Teoría que coincide con varios autores como (Chaves, 2019), (Garcia-Gaitero, Garcia-Gaitero, \& Martin-Martin, 2018) y (Bravo, Intriago, Holguín-Villafuerte, GarzonMolina, \& Arcia-Ortega, 2017), quienes afirman que el uso de juegos motiva en aprendizaje del inglés en los diferentes niveles de educación, sobre todo con niños.

Todos los resultados obtenidos son favorables en el proceso educativo, debido a que los estudiantes con la nueva estrategia cambian su forma de percibir al inglés y lo adoptan como una asignatura de gran importancia dentro del currículo de estudios, con la aplicación de ésta didáctica se motivó al estudiante a involucrarse en el proceso pedagógico y de esta manera obtener conocimientos significativos. Tal cual lo manifiesta (Gardner, 1985), la motivación juega un papel importante dentro del aprendizaje de un idioma, lo cual concuerda con los resultados obtenido que demuestran que con el uso de juegos se han motiva a la adquisición del idioma. 


\section{PROPUESTA}

En vista de que los resultados motivaron el aprendizaje del Inglés con la aplicación de la gamificación se propone 3 aspectos a desarrollarse con la aplicación de la plataforma educativa Mobbyt como son: Planificación, Implementación y Evaluación, con el fin de promover la concentración, memorización, asimilación y procesamiento del léxico y estructuras sintácticas del idioma extranjero.

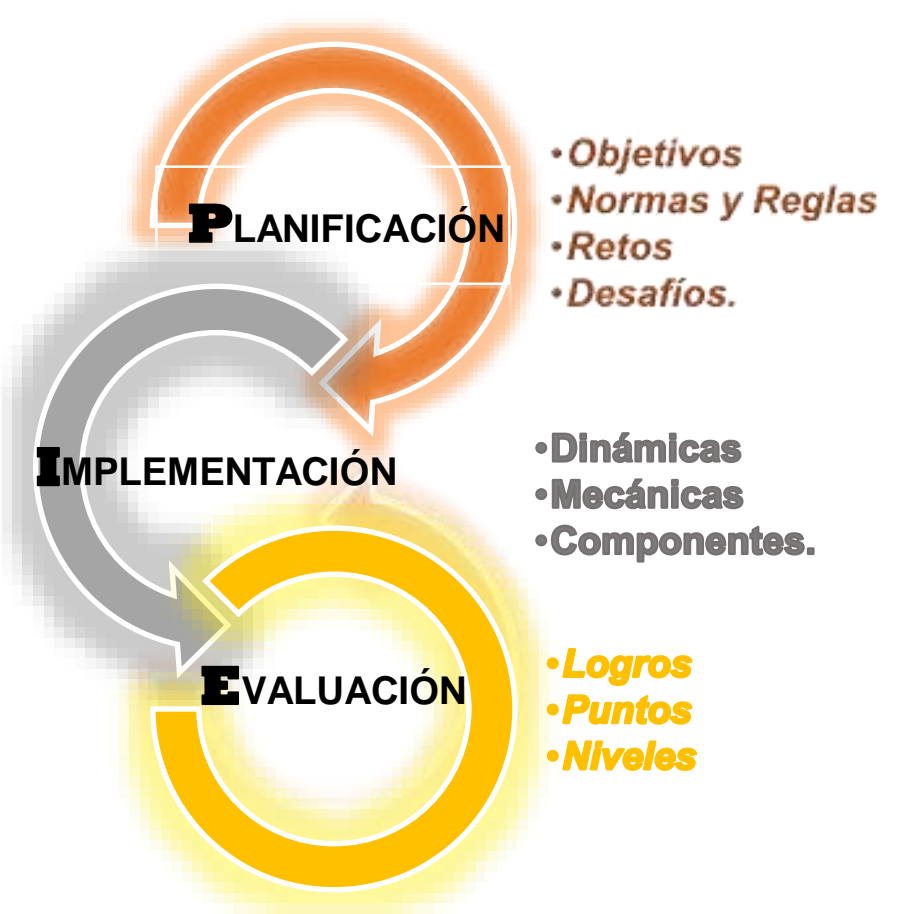

Figura 1. Actividades plataforma educativa Mobbyt. Fuente: Elaboración propia

1. Planificación: La gamificación como estrategia metodológica permite al estudiante aprender en un ambiente lúdico, mismo que propicia motivación e interés por el aprendizaje. Los elementos a consider dentro de una planificación son Objetivos, normas y reglas, retos y desafíos.

- Objetivos: Lo que desea alcanzar con los estudiantes a través de la gamificación, para ello se debe considerar aspectos y temáticas del currículo que permitan 
gamificarlo y de esta manera lograr que los estudiantes sean capaces de tomar decisiones, asumir nuevos retos, ser reconocido por sus logros mientras adquiere conocimientos nuevos dentro de este proceso.

- Normas y reglas: Establecer normas y reglas dentro del juego es importante para cada uno de los estudiantes dándoles a conocer limitaciones, pasos 0 especificaciones a seguir dentro de este proceso. Todas las reglas establecidas ayudarán a promover un comportamiento adecuado, suscita una competición limpia y sobre todo origina una participación activa.

- Retos: Al ejecutar el juego por primera vez es importante definir lo que se tiene que hacer para lograrlo, el reto debe ser concreto y motivador, debe ser explicado al inicio, durante y después del juego.

- Desafíos: se refiere a la competencia entre jugadores para obtener un puntaje dentro la actividad planteada, donde el estudiante con mayor puntaje es el que recibe la recompensa.

2. Implementación: La gamificación al ser considerada una didáctica lúdica permite transformar el aprendizaje de capacidades y conocimientos en juego. Además, contribuyen al desarrollo de competencias en el proceso de enseñanza aprendizaje y promueve conductas positivas en cada uno de los participantes. Los elementos considerados dentro de la implementación son: dinámicas, mecánicas y componentes.

- Las dinámicas: son el motor que permite el funcionamiento de la estrategia lúdica, están directamente relacionado con el desempeño de los participantes. El propósito de las dinámicas de juego es lograr experiencias de aprendizaje que fomentan la interacción, la autonomía y el trabajo en equipo de los participantes, también permiten cumplir los objetivos planteados y alcanzar metas propuestas.

- La mecánica: está relacionada con la motivación y el comportamiento de cada uno de los educandos. Dentro de la mecánica del juego encontramos a las reglas y recompensa que hacen que los juegos se conviertan en desafíos provocando emociones placenteras y satisfactoria. 
- Los componentes: son herramientas y recursos que se utilizan dentro las actividades lúdicas.

3. Evaluación: Determina el grado de alcance de los objetivos y la eficacia de la actividad o el sentimiento de bienestar que el estudiante siente al concluir un juego dentro del proceso educativo. Al combinar juego con aprendizaje se deber utilizar un sistema de recompensa y de esta manera se mantiene la atención del educando al reconocer sus logros, mismos que lo animaran a continuar preparándose. Sin embargo, hay que mantener un equilibrio porque demasiados premios materiales pueden restar relevancia y llegar a ser contraproducente. La evaluación consta de logros, puntos niveles.

- Logros: dentro de la gamificación es importante establecer puntaciones o premios por los logros obtenidos, valorando no solo la adquisición de contenidos sino también el comportamiento y capacidad de trabajo individual y grupal.

- Puntos: permiten retroalimentar a los jugadores para mejorar habilidades y destrezas e incentivar a continuar con este proceso. Hay 5 tipos de sistema de puntos:

Puntos de experiencia: son los que realizan un seguimiento de las experiencias adquiridas por el jugador.

Puntos canjeables: son los puntos que se cambian por realizar ciertas actividades. Reputación: son los puntos que otorgan un estatus al participante.

Habilidad: son los puntos que permiten demostrar la capacidad del estudiante en cierta área.

Karma: puntos extras que se obtienen y de esta manera ayudar a los demás.

- Niveles: El juego se basa en el equilibrio entre dificultad de un reto y satisfacción que se obtiene al superarlo. Es importante tomar en cuenta el avance del estudiante y la práctica que lo realiza, para de esta manera aumentar la dificultad y de esta manera se adapte al dominio que se ha ido adquiriendo. De este modo se mantendrá la tensión reto-superación sobre todo la motivación del estudiante para continuar aprendiendo a través del juego. 


\section{CONCLUSIONES}

La problemática sobre el aprendizaje del inglés en educación media es de gran importancia, por cuanto la asignatura que es parte del currículo y debe ser aprobada para pasar al próximo nivel, sin embargo, a pesar de la obligatoriedad a los estudiantes no les gusta la asignatura porque no se ha utilizado didácticas motivadoras que despierten el interés por el aprendizaje de la lengua extranjera.

Al implementar la gamificación como una estrategia didáctica que despierte el interés por el aprendizaje del idioma extranjero, los estudiantes se motivaron a adquirir conocimientos nuevos del inglés mismos que lo utilizan en su vida cotidiana, didáctica de gran importancia para varios autores, quienes afirman la efectividad del uso de estrategias motivacionales en el aula que mejoran el nivel de suficiencia del inglés, sin embargo hay que analizar las desventajas que este puede provocar.

Con el resultado obtenido sobre el método preferido para aprender una nueva lengua se hace indispensable reflexionar que los métodos tradicionales de repetición y memorización pasan a ser obsoletos, puesto que los estudiantes actuales necesitan métodos innovadores que con la ayuda de las TIC faciliten adquirir conocimientos que puedan utilizar en su vida cotidiana.

Indudablemente los docentes juegan un papel preponderante en el proceso de aprendizaje, por cuanto ellos son los encargados de proponer estrategias que motiven al estudiante a aprender. Con la implementación de actividades lúdicas en el proceso pedagógico, el docente impulsa a estudiante a ser un ente activo y participativo ayudándole a adquirir un aprendizaje de forma efectiva.

Para concluir se puede acotar que el uso de la gamificación motiva al estudiante a involucrarse de una forma dinámica en el aprendizaje, adoptándole como una didáctica innovadora que facilita el análisis y adquisición de contenidos de una forma significativa.

\section{FINANCIAMIENTO}

No monetario. 


\section{AGRADECIMIENTO}

A todo el talento humano de la Unidad Educativa Particular La Providencia de la ciudad de Azogues, provincia del Cañar, apoyar el desarrollo de la investigación.

\section{REFERENCIAS CONSULTADA}

Bravo, J. C., Intriago, E. A., Holguín-Villafuerte, J., Garzon-Molina, G., \& Arcia-Ortega, L. (14 de 1 de 2017). Motivation and Autonomy in Learning English as Foreign Language: A Case Study of Ecuadorian College Students [Motivación y autonomía en el aprendizaje del inglés como lengua extranjera: un estudio de caso de estudiantes universitarios ecuatorianos]. English Language Teaching, Vol. 10, No. 2. Obtenido de https://n9.cl/cyo4a

Briceño-de-Osorio, A. (2019). Aprendizaje de lenguas para la diversidad cultural. [Language learning for cultural diversity]. EPISTEME KOINONIA, 2(4), 5371. http://dx.doi.org/10.35381/e.k.v2i4.524

Cedeño Macías, L. M. (2017). Cybertesis. Obtenido de https://n9.cl/3fim

Caicedo-Coello, J., Vallejo-Valdivieso, P., \& Moya-Martínez, M. (2020). Juegos dirigidos y la motivación en estudiantes del décimo año de Educación General Básica. [Directed games and motivation in students of the tenth year of Basic General Education]. Revista Arbitrada Interdisciplinaria Koinonía, 5(9), 189-203. doi:http://dx.doi.org/10.35381/r.k.v5i9.617

Chaves, Y. B. (2019). Revisión de experiencias de gamificación en la enseñanza de lenguas extranjeras [Review of gamification experiences in teaching foreign languages]. ReiDoCrea, 422-430. Obtenido de Reidocrea | ISSN: 2254-5883 | Volumen 8. Articulo 33. Páginas 422-430 422: https://n9.cl/j9f0

Chávez Zambano Mirian, S. V. (2017). La importancia del aprendizaje y conocimiento del idioma inglés en la enseñanza superior [The importance of learning and knowing the English language in higher education]. Dominio de las Ciencias, 3, 759-771. doi:http://dx.doi.org/10.23857/dc.v3i3\%20mon.707

Cordero-Naspud, E. I., Erazo-Álvarez, J. C., Narváez-Zurita, C. I., \& Cordero-Guzmán, D. M. (2020). Soluciones corporativas de inteligencia de negocios en las pequeñas y medianas empresas [Corporate business intelligence solutions in small and medium enterprises]. Revista Arbitrada Interdisciplinaria Koinonía, 483-513. doi:http://dx.doi.org/10.35381/r.k.v5i10.703 
Deterding, S. D. (2011). From game design elements to gamefulness: defining "gamification” [Desde los elementos de diseño del juego hasta el juego: definición de "gamificación"]. MindTrek, 9-15. doi:https://doi.org/10.1145/2181037.2181040

Fernandez- Solo de Zaldivar, I. (2015). Centro de Comunicacion y Pedagógica [Communication and Pedagogical Center]. Obtenido de http://www.centrocp.com/

Figeroa-Flores, J. F. (2015). Using Gamification to Enhance Second Language Learning [Uso de la gamificación para mejorar el aprendizaje de un segundo idioma]. Digital Education Review, 32-54. Obtenido de https://n9.cl/fsvz

Garcia-Gaitero, F., Garcia-Gaitero, O., \& Martin-Martin, M. (2018). La Gamificación como Recurso para la Mejora del Aprendizaje del Inglés en Educación Primaria [Gamification as a Resource for Improving English Learning in Primary Education]. Rilme, 466-468. Obtenido de https://n9.cl/wd5ea

Gardner, R. C. (1985). The role of attitude and motivation [The role of attitude and motivation]. London: Edward Arnold. Obtenido de https://n9.cl/ilxh

Johnson, L., Becker, A. S., Cummins, M., Estrada, V., Freman, A., \& Hall, C. (2016). NMC Informe Horizon [NMC Horizon Report]. Obtenido de https://n9.cl/lese

Llorens, F., Largo, F., Gallego Duran, J. C., Campañ Rosique, P., Satorre Cuerda, R., \& Molina Carmona, R. (Marzo de 2016). Gamification of the learning process: lessons learned [Gamificación del proceso de aprendizaje: lecciones aprendidas]. Revista Iberoamericana de Tecnologías del Aprendizaje, 4(1), 227 - 234. doi:10.1109 / RITA.2016.2619138

Marín Díaz, V. (2015). La Gamificación educativa. Una alternativa para la enseñanza creativa [The educational gamification. An alternative to creative teaching]. Digital Education Review, 1-8. Obtenido de https://n9.cl/p1qn2

Medina-Chicaiza, R., \& Sanchéz Quishpe, M. B. (2018). Gamificación: una estrategia para la adquisición de vocabulario del idioma Inglés nivel pre-intermedio [Gamification: a strategy for acquiring English language vocabulary at the preintermediate level]. Quito: Pontificia Universidad Católica. Obtenido de https://n9.cl/f1mu3

Ministerio de Educacación Ecuador. (2016). Currículo 2016-Lengua Extranjera [Curriculum 2016-Foreign Language]. Obtenido de https://n9.cl/4pbm 
Ortega Auquilla, D., Hidalgo Camacho, C., Siguenza Garzón, P., \& Cherres Fajardo, S. (2020). La Motivación como factor para el aprendizaje del idioma inglés en el contexto universitario ecuatoriano [Motivation as a factor for learning the English language in the Ecuadorian university context]. Publicando, 9-20. Obtenido de https://n9.cl/oj4iq

Ortega-Auquilla, D. P., \& Auccahuallpa-Fernandez, R. (2017). La Educacion Ecuatoriana en Inglés:Nivel de Dominio y Competencias Linguisticas de los Estudiantes Rurales [Ecuadorian Education in English: Level of Mastery and Linguistic Competences of Rural Students]. Revista Scientific-Articulo Arbitrado, 52-73. doi:https://doi.org/10.29394/scientific.issn.2542-2987.2017.2.6.3.52-73

Ortiz, A.-M., Jordán, J., \& Agredal, M. (2018). Gamificación en educación: una panorámica sobre el estado de la cuestión [Gamification in education: an overview of the state of affairs]. Revista da Faculdade de Educação da Universidade de São Paulo, 44, 27-38. Obtenido de https://n9.cl/io2x

Richards Jack C., R. T. (1986). Approaches and Methods in Language Teaching [Approaches and Methods in Language Teaching]. United Kinddom.: Cambridge University Press. Obtenido de https://n9.cl/fzo8p

Ruiz de la Hermosa, L. (2016). Unir Gamificación y Experiencia de Usuario para mejorar la experiencia docente [Unite Gamification and User Experience to improve the teaching experience]. Revista Iberoamericana de Educación a Distancia, vol. 19(núm.), 125-142. Obtenido de https://n9.cl/5kwf

Sanguineti, J. J. (2014). Neurociencia y filosofia del hombre [Neuroscience and philosophy of man]. Buenos Aires: Palabra. Obtenido de https://n9.cl/lwb6c

Vanzin, T., Batista, C. R., \& Lopes da Silva, A. R. (2013). Contribuições da criatividade em diferentes áreas do conhecimento [Contributions of creativity in different areas of knowledge]. Rio de Janiero: Pimenta Cultural. Obtenido de https://n9.cl/uy89 